\title{
The Performance of a Scale-Aware Nonlocal PBL Scheme for the Subkilometer Simulation of a Deep CBL over the Taklimakan Desert
}

\author{
Hongxiong $X u\left(\mathbb{D},{ }^{1,2,3}\right.$ Yinjun Wang $\mathbb{D},,^{1,2,3}$ and Minzhong Wang $\mathbb{D}^{1,2,3}$ \\ ${ }^{1}$ State Key Laboratory of Severe Weather, Chinese Academy of Meteorological Sciences, Beijing 100081, China \\ ${ }^{2}$ Institute of Desert Meteorology, CMA (Chinese Meteorological Administration), Urumqi 830002, China \\ ${ }^{3}$ Taklimakan Desert Atmospheric Environment Observation Experimental Station, Tazhong 841000, China
}

Correspondence should be addressed to Yinjun Wang; pbl_wyj@sina.cn

Received 5 October 2017; Revised 2 January 2018; Accepted 24 January 2018; Published 27 February 2018

Academic Editor: Rossella Ferretti

Copyright (C) 2018 Hongxiong Xu et al. This is an open access article distributed under the Creative Commons Attribution License, which permits unrestricted use, distribution, and reproduction in any medium, provided the original work is properly cited.

\begin{abstract}
Although realistic representation of the convective boundary layer (CBL) in the desert region in Northwest China is important for weather forecasts and climate simulations, evaluations of the performance of various planetary boundary layer (PBL) schemes in simulating the CBL in the region are rare. In this study, the performance of a scale-aware PBL scheme newly implemented into the Weather Research and Forecasting (WRF) model in simulating the CBL in the Taklimakan desert is evaluated based on a comparison with both the WRF-LES simulations and observations, with the focus on scale dependencies of the simulations compared to the conventional PBL scheme. A series of simulations are performed with a scale-aware PBL scheme (Shin-Hong) and the conventional PBL scheme (YSU) for a deep CBL observed at Tazhong station in the central Taklimakan on 1 July 2016. The CBL was over $5000 \mathrm{~m}$ deep with wider and deeper rolls than in a shallow boundary layer. The results showed that the vertical structure simulated with the Shin-Hong scheme was closer to that in both the WRF-LES (large-eddy-simulation) and observations than that simulated with the YSU. The simulation with the scale-aware scheme reproduced cellular rolls similar to those in the WRF-LES, while the conventional PBL scheme struggled to trigger intense convective cells rather than cellular rolls. The results strongly suggest that the scale-aware nonlocal PBL scheme can be used to adequately reproduce the scale and evolution of the observed rolls in the deep CBL in Taklimakan desert at subkilometer resolutions.
\end{abstract}

\section{Introduction}

The Taklimakan desert is a key component of the earth's climate system. Owing to the extremely high near-surface temperatures in summer, the boundary layer in Taklimakan desert is commonly convective and reaches $5000 \mathrm{~m}$ height, making it one of the deepest PBLs on the earth. The deep CBL, which is significantly higher than that of the surrounding mountains and oases, may cause various degrees of influence on the regional atmospheric circulation, weather, and climate [1]. Accurately simulating the planetary boundary layer (PBL) processes within the Taklimakan desert is critical for correctly simulating the weather of its surrounding areas.

The representation of convective boundary layer (CBL) turbulence by numerical models is either completely parameterized, such as in mesoscale or global circulation models, or explicitly simulated as in cloud-resolving models or largeeddy simulations. These two completely different modeling strategies are selected based on the typical grid spacing of the model with respect to the dominant length scale of the flow [2]. Mesoscale atmospheric models are still not fine enough to explicitly resolve all relevant scales of atmospheric motions whose effects must be included in the NWP models to reasonably predict atmospheric states [3]. Turbulent motions belong to these subgrid-scale motions and can significantly alter the atmospheric status by means of turbulent mixing, predominantly throughout the planetary boundary layer (PBL) $[4,5]$. Limited by coarse vertical and time resolution, planetary boundary layer (PBL) parameterizations in mesoscale models have been developed for horizontal resolutions that cannot resolve any turbulence in the PBL [6]. 
Thanks to recent increasing in computing power, numerical weather prediction models can now run at very fine grid sizes. However, turbulence is well represented by atmospheric models at very fine grid sizes, from 10 to $100 \mathrm{~m}$ [7-10], for which turbulent movements are mainly resolved, and by atmospheric models with grid sizes greater than $2 \mathrm{~km}$, for which those movements are entirely parameterized [11-14]. But what happens at intermediate scales? [15]. Resolution from $1 \mathrm{~km}$ to $100 \mathrm{~m}$ is termed "terra incognita" $[2,16]$ or "gray zone." Difficulties arise in this region, since the model grid spacing is comparable to the dominant length scale of the flow. The dominant flow feature is only barely or not at all resolvable on a gray zone grid [2]. The terra incognita has traditionally been treated as a numerical difficulty related to the subgrid turbulence parameterization.

A few recent studies examined resolutions of the problem in gray zone Shin and Hong [4], Honnert et al. [15], and Shin and Hong [17]. Wyngaard [16] suggested a tensor instead of a scalar representation of the eddy diffusivity to improve gray zone simulations using Eddy Viscosity closures. Honnert et al. [15] indicated the importance of accurately representing SGS thermals by taking grid size into account when developing a SGS turbulence model for the gray zone resolutions. Shin and Hong [17] separately analyzed the gridscale dependencies of nonlocal and local transports. The separate treatment is meaningful, considering that nonlocal and local parts are separately parameterized in NWP models and GCMs. As in Shin and Hong [4], the SGS local transport is formulated by multiplying a grid-size dependency function with the total local transport profile, which is calculated using an eddy diffusivity formula. The new PBL scheme, Shin-Hong scheme, which is mainly based on conventional nonlocal PBL scheme with scale dependent, has been added to WRF. Their further study suggests that ideal CBL cases, by considering the scale dependency in the parameterized vertical heat transport, improvements over the conventional nonlocal K-profile (e.g., YSU) model appear in mean profiles, resolved and SGS vertical transport profiles with their gridsize dependency, and the energy spectrum.

However, the Shin-Hong parameterization scheme has not been developed for extreme conditions like Taklimakan with super high PBL depth, where their assumptions and reference data from LES solutions of domains were only extended to $4000 \mathrm{~m}$ [17]. Direct applications of the existing scale-dependent $\mathrm{PBL}$ parameterizations to deep $\mathrm{CBL}$ (>5000 $\mathrm{m}$ ) over desert such as Taklimakan may be questionable. Thus, the performance of the Shin-Hong scheme in the WRF model over deep CBL over Taklimakan desert needs to be further evaluated.

In this study, WRF version 3.8.1 is used to simulate CBL of the Taklimakan on 1 July 2016, during a field observation experiment. The field observation experiment was held during the month of July 2016 in Tazhong station, which is located at the center of Taklimakan, by the Institute of Desert Meteorology (IDM), Chinese Meteorological Administration (CMA), Urumqi $[1,18,19]$, with a GPS sounding system, an eddy correlation measurement system, and a radiation measurement system as the main equipment. The eddy correlation system set up at a height of $10 \mathrm{~m}$ was a R3-50 supersonic anemometer developed by Gill Company, UK. The data acquisition frequency was $20 \mathrm{~Hz}$. GPS sounding system was developed by the Institute of China Aerospace Science \& Industry Corp. (CASIC23) number 23. Observations collected during this experiment provide a comprehensive validation dataset for model experiments. The sensitivities of the WRF simulations to the use of two PBL schemes, the YSU scheme (scale independent) and Shin-Hong scheme (scale dependent), as well as the WRF-LES in real case simulation, are examined. We identify the impact of scale-dependent PBL scheme on model performances with possible consequences for Taklimakan desert CBL simulation and explore the causes of those impacts.

\section{The Model and Setup}

The WRF model [20], version 3.8.1, was utilized in this study to perform subkilometer resolution simulation for the deep CBL event which occurred in Taklimakam desert on 1 July 2016. Figure 1 shows the model domain and the nested meshes for our experiments. The outermost domain (d01) had the horizontal grid spacing of $12 \mathrm{~km}$ with $411 \times 321$ grid points and three one-way nested meshes (d02, d03, and d04) had horizontal grid spacing of $3 \mathrm{~km}, 1 \mathrm{~km}$, and $0.33 \mathrm{~km}$ with their corresponding grid points of $791 \times 651,211 \times 201$, and $403 \times$ 406, respectively (Figure 1). All meshes had 51 vertical levels extending from the surface to $50 \mathrm{hPa}$. Heights for lowest 10 levels are $1130.4,1157.6,1207.6,1294.5,1423.6,1591.5,1795.0$, 2021.2, 2271.5, and $2557.5 \mathrm{~m}$.

The model physics options include the WSM5 microphysics scheme [21], the Yonsei University (YSU) PBL scheme [22], Revised MM5 Monin-Obukhov surface layer scheme [23], the Kain-Fritsch cumulus parameterization scheme [24, 25], the Noah land surface model [26, 27], the Rapid Radiative Transfer Model [28] longwave, and the Dudhia shortwave radiation scheme [29]. The cumulus parameterization scheme was only applied to the d01 $(12 \mathrm{~km})$ domain to parameterize the subgrid-scale convective rainfall. The largeeddy-simulation (LES) is only applied to the innermost highest resolution $\mathrm{d} 04(0.333 \mathrm{~km})$.

The initial and lateral boundary conditions were obtained from the National Centers for Environmental Predictions (NCEP) Global Data Assimilation System (GDAS) Final Global Analyses (FNL), which has horizontal resolution of 0.25 degrees by 0.25 degrees at every $6 \mathrm{~h}$ intervals with data available at the surface and 26 pressure levels from 1000 to $10 \mathrm{hPa}$ [30]. The model was initialized at $0800 \mathrm{BJT}, 1$ July 2016, and integrated for $12 \mathrm{~h}$. Three experiments were performed with different PBL schemes: one with the WRF-LES settings, one with the YSU scheme, and one with the scale-aware ShinHong scheme.

\section{The PBL Parameterization}

3.1. The YSU PBL Scheme. The YSU [21] PBL scheme is the first-order closure nonlocal scheme, since it does not require any additional prognostic equation for turbulent kinetic energy to represent the effects of turbulence on mean 


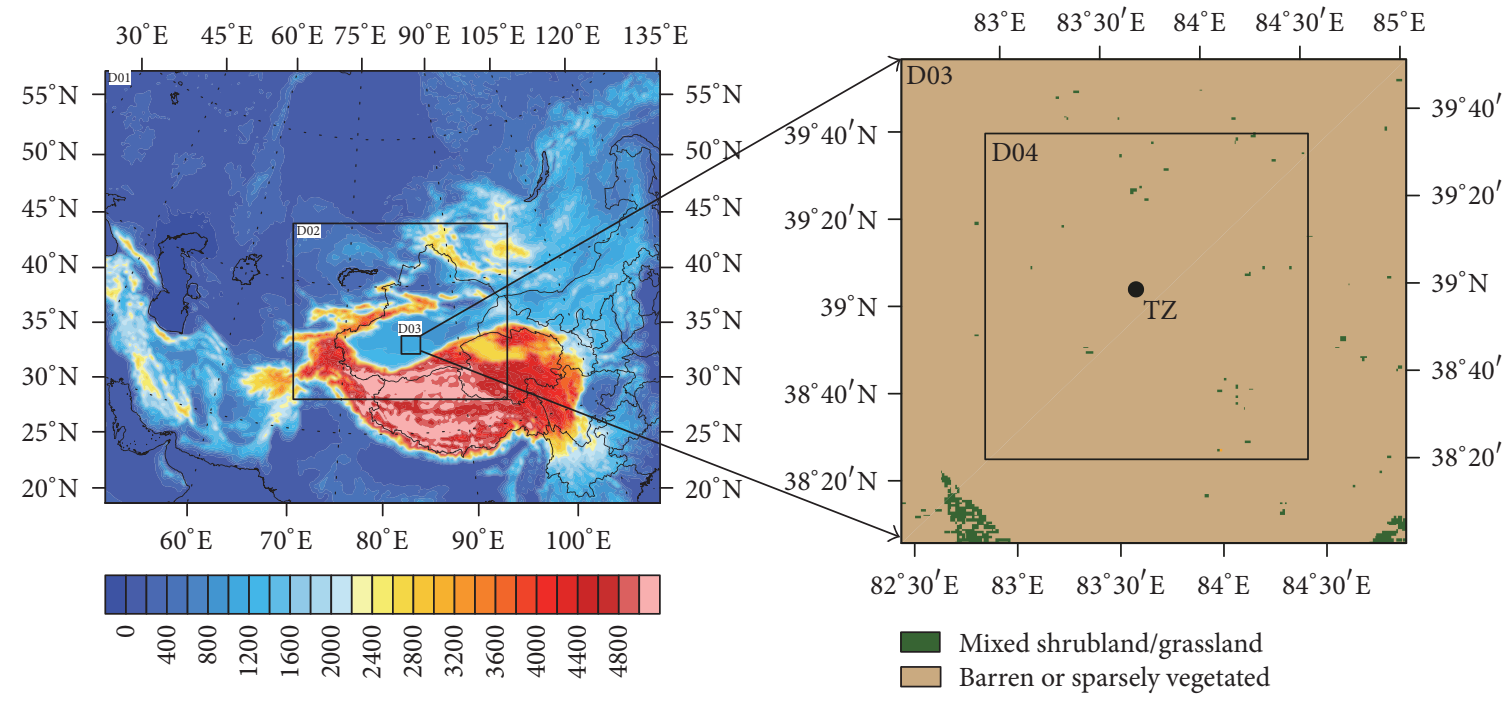

(a)

(b)

FIGURE 1: Simulation domains used in ARW model with terrain height (shading, units: m); (b) land use categories for domains D03 and D04.

variables. The vertical diffusion of a variable $C$ can be written as

$$
\frac{\partial C}{\partial t}=\frac{\partial}{\partial z}\left[K_{c}\left(\frac{\partial C}{\partial z}-\gamma_{c}\right)-\overline{\left(w^{\prime} c^{\prime}\right)_{h}}\left(\frac{z}{h}\right)^{3}\right]
$$

where $K_{c}$ is the eddy diffusivity coefficient, $z$ is the height, and $t$ is time. The first term on the right hand side of (1) is the traditional vertical diffusion term that includes the nonlocal mixing by convective eddies. $\gamma_{c}$ is a countergradient transport term, which incorporates the contribution of convective eddies to the total flux. In the second term, $\overline{\left(w^{\prime} c^{\prime}\right)_{h}}$ is the turbulent flux associated with the detrainment at the inversion base. The YSU scheme uses the $K$ profile in determining the diffusivity $K_{c}$ within the boundary layer. The local momentum eddy diffusivity is computed from

$$
K_{m}=k w_{s} z\left(1-\frac{z}{h}\right)^{p}
$$

where $p$ is the profile shape exponent taken to be $2, k$ is the von Kármán constant $(0.4), w_{s}$ is the mixed layer velocity scale, and $h$ is the height of the PBL.

3.2. The Shin-Hong Scheme. When the subgrid and resolved parts are studied, the dimensionless variables determined by the similarity theorem are added to the Deardorff free convective scaling variables. Similarity functions for the subgrid and resolved parts are assumed to be the product of the similarity function of the total variables and a "partial" similarity function according to the grid size. To determine the partial similarity function form, the grid-size dependency functions are fitted to the reference data from the LES. The resolved and subgrid parts of the variables at coarser grid sizes are then deduced from the LES fields. For Shin-Hong PBL scheme [2], the original forms of the functions suggested by Talbot et al. [3] are maintained, and only the constants are tuned to minimize the root-mean-square error between the reference data and simple functions.

The Shin-Hong PBL scheme is a scale-aware scheme. The SGS transport profile is parameterized based on the 2013 conceptual derivation documented in Shin and Hong [17]. First, the nonlocal transport by strong updrafts and local transport by the remaining small-scale eddies are separately calculated. Second, the SGS nonlocal transport is formulated by multiplying a grid-size dependency function with the total nonlocal transport profile fitted to the LES output as given below

$$
\begin{aligned}
& \left\langle w^{\prime} \theta^{\prime}\right\rangle^{S\left(\Delta_{*}\right), \mathrm{NL}}=\left\langle w^{\prime} \theta^{\prime}\right\rangle^{\mathrm{NL}} P_{\mathrm{NL}}\left(\Delta_{* \mathrm{CS}}\right) \\
& P_{\mathrm{NL}}\left(\Delta_{* \mathrm{CS}}\right) \\
& =0.243 \frac{\left(\Delta_{* \mathrm{CS}}\right)^{2}+0.936\left(\Delta_{* \mathrm{CS}}\right)^{7 / 8}-1.110}{\left(\Delta_{* \mathrm{CS}}\right)^{2}+0.312\left(\Delta_{* \mathrm{CS}}\right)^{7 / 8}+0.329} \\
& \quad+0.757,
\end{aligned}
$$

where the angle brackets refer to a domain average and the superscripts $S$ and NL designate SGS and nonlocal, respectively. The term $P_{\mathrm{NL}}\left(\Delta_{* \mathrm{CS}}\right)$ is the grid-size dependency function of the SGS nonlocal heat transport from an empirical function fitted to the reference data for a free-convection case. $\Delta_{*}=\Delta / h$, and CS is a stability function. Finally, the SGS local transport is formulated by multiplying a grid-size dependency function with the total local transport profile, which is calculated using an eddy diffusivity formula.

$$
\begin{aligned}
\left\langle w^{\prime} \theta^{\prime}\right\rangle^{S\left(\Delta_{*}\right), L} & =-K_{H} P_{L}\left(\Delta_{*}\right) \frac{\partial \bar{\theta}^{\Delta}}{\partial z} \\
P_{L}\left(\Delta_{*}\right) & =0.280 \frac{\left(\Delta_{*}\right)^{2}+0.870\left(\Delta_{*}\right)^{1 / 2}-0.913}{\left(\Delta_{*}\right)^{2}+0.153\left(\Delta_{*}\right)^{1 / 2}+0.278}
\end{aligned}
$$




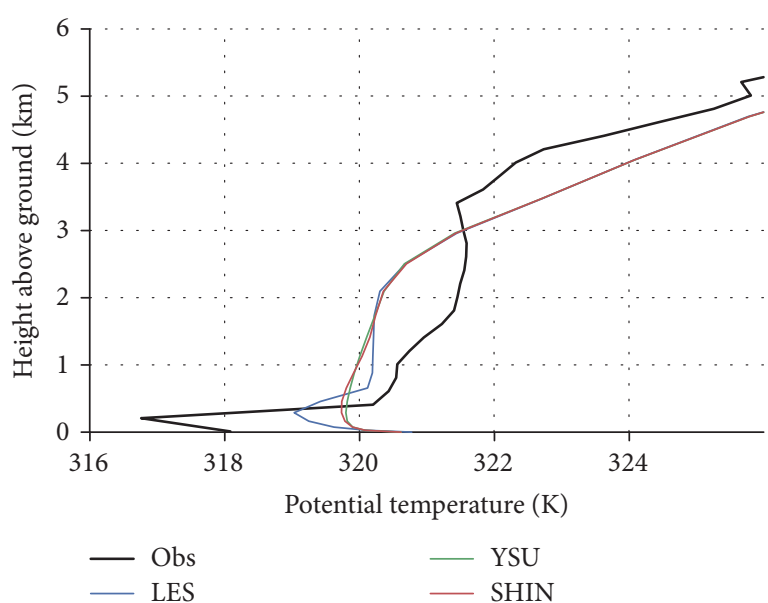

(a)

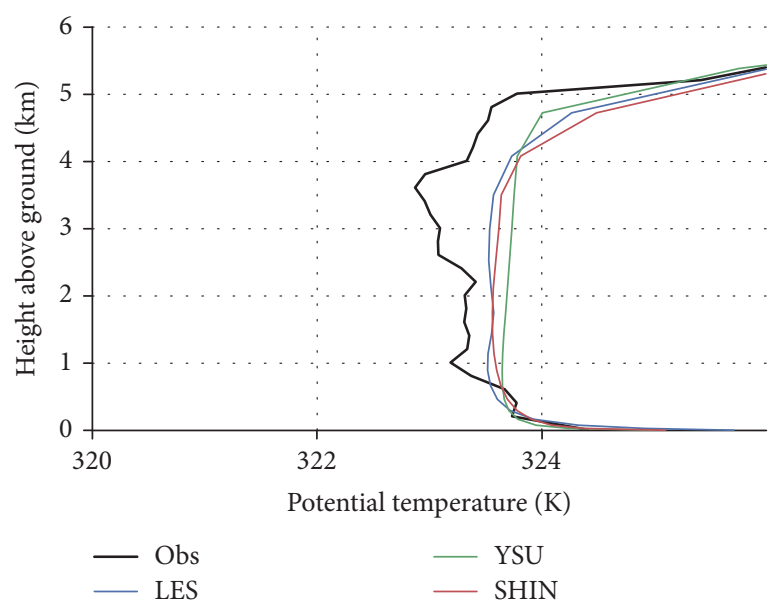

(c)

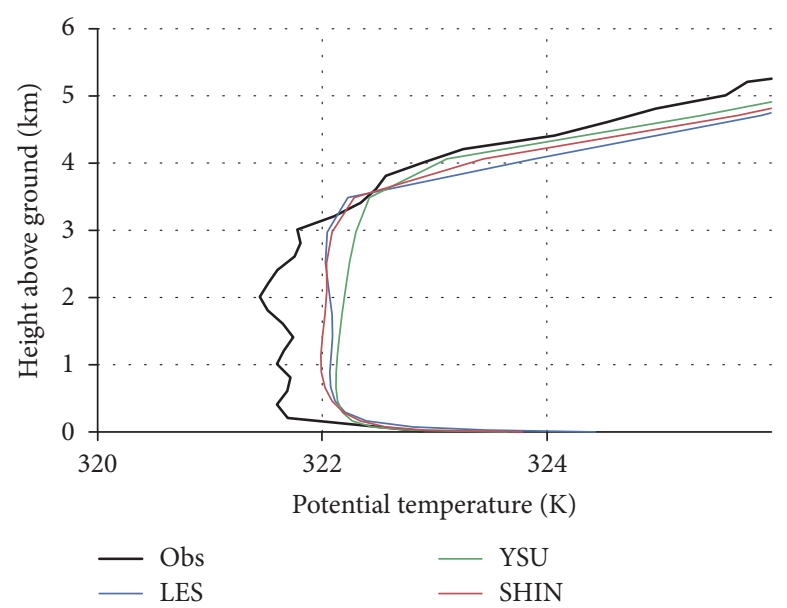

(b)

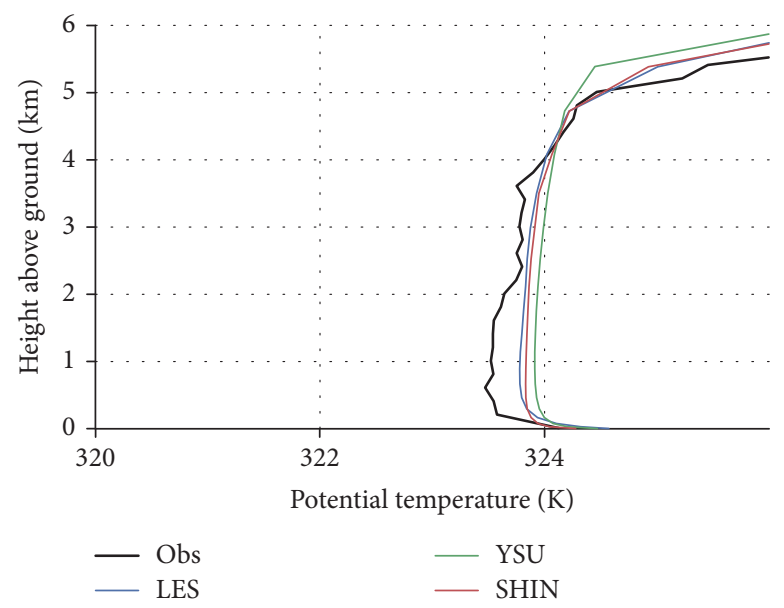

(d)

FIGURE 2: Vertical profiles of potential temperature (units: K) from simulations and observation of GPS sounding at Tazhong station (83.63 ${ }^{\circ} \mathrm{E}$, $39.03^{\circ} \mathrm{N}$ ) at (a) 1100, (b) 1400, (c) 1700, and (d) 2000 BJT, 1 July 2016.

where the angle brackets refer to a domain average as in (3) and the superscripts $S$ and $L$ designate SGS and local, respectively. The term $P_{L}\left(\Delta_{*}\right)$ is the grid-size dependency function of the SGS local heat transport from an empirical function fitted to the reference data for a free-convection case and is considered in computing the vertical diffusivity $\left(K_{H}\right)$ to suppress the parameterized transport as grid size decreases. The main improvements of the Shin-Hong PBL PBL scheme are the prescribed nonlocal heat transport profile fitted to the LES output and the inclusion of an explicit scale dependency function for vertical transport in CBL [4]. Thus, the Shin-Hong PBL parameterization is a scale-aware scheme and works at the gray zone resolution for CBL.

\section{Results}

4.1. The Boundary Layer Structures. The vertical profiles of the simulated potential temperature and water vapor mixing ratio are compared with the GPS radio-sounding data at Tazhong station in Figures 2 and 3, respectively. All simulations initially reproduced a cooler PBL than that observed, due to the underestimated RL (residual layer) in the initial conditions, all model simulations nearly the same, but drier and cooler than measures of 1100 BJT, 1 July, at height from 2 to $3.5 \mathrm{Km}$ (Figures 2(a) and 3(a)). The observed potential temperature profile is strongly unstable in the CBL (Figure 2(a)) at 1100 BJT, 1 July 2016. The WRFLES experiment simulated the unstable profile at nearly the same altitude as in observations but with a smaller lapse rate. The YSU and Shin-Hong experiments produced very weak unstable profiles, with the $\theta$ profiles quite different from those in observations and the WRF-LES experiment. However, all model simulations produced a CBL warmer than that observed (Figure 3(b)) at 1400 BJT, 1 July. Note that the largest biases in potential temperature and PBLH (Figure 2) were from the YSU experiments, followed by the Shin-Hong experiment, and then the WRF-LES. This suggests that the scale-aware scheme performed better than the traditional scheme. Furthermore, all simulations produced too large heating rate in the morning, due to large discrepancies in surface sensible heat flux (SH) between the model and the observation (not shown). In the afternoon, the model 


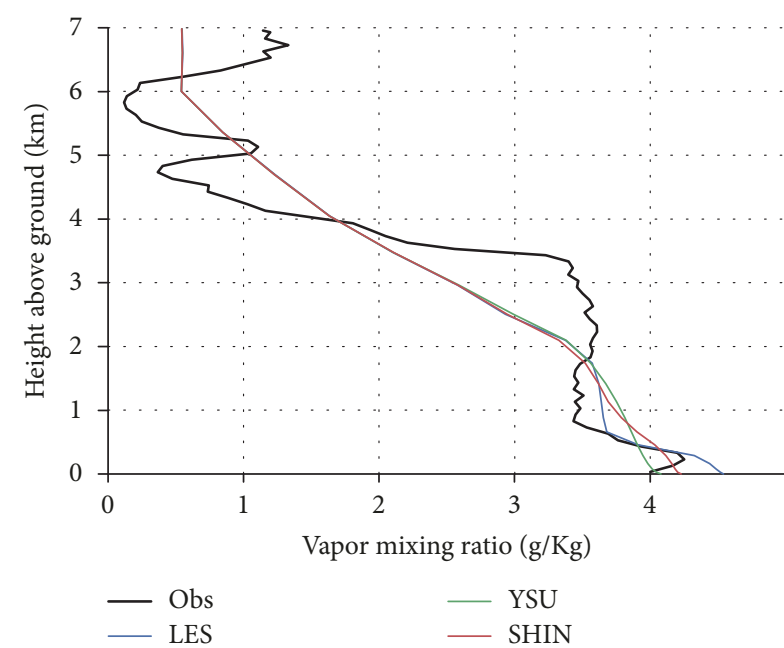

(a) 1100 BJT, 1 July 2016

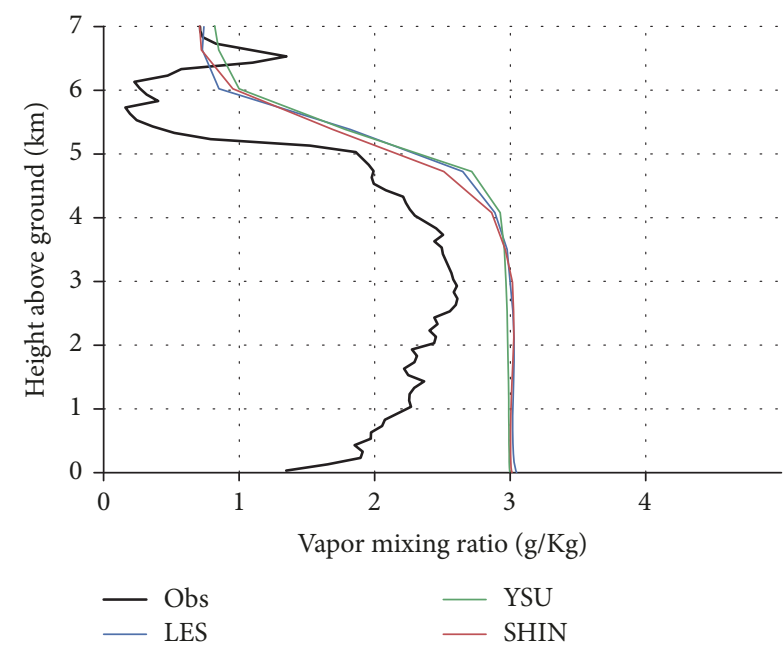

(c) 1700 BJT, 1 July 2016

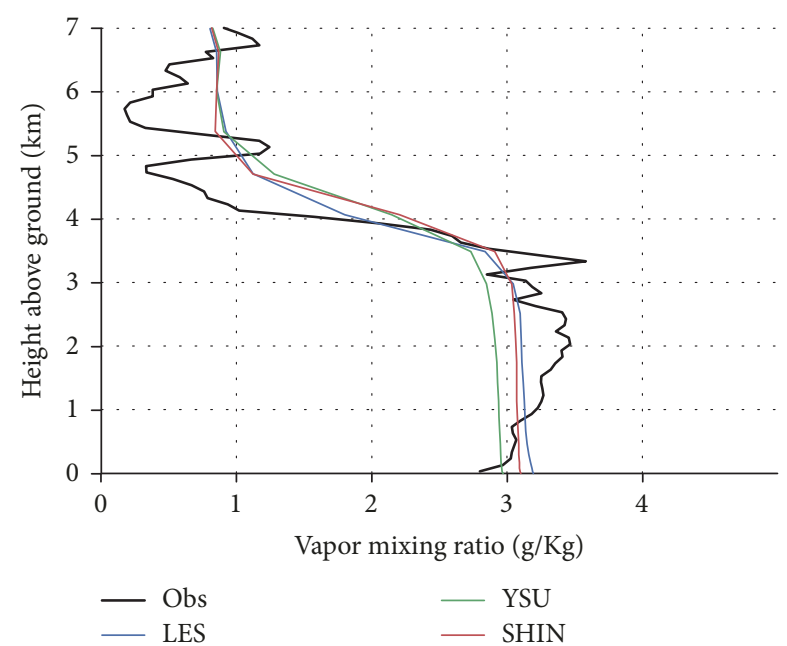

(b) 1400 BJT, 1 July 2016

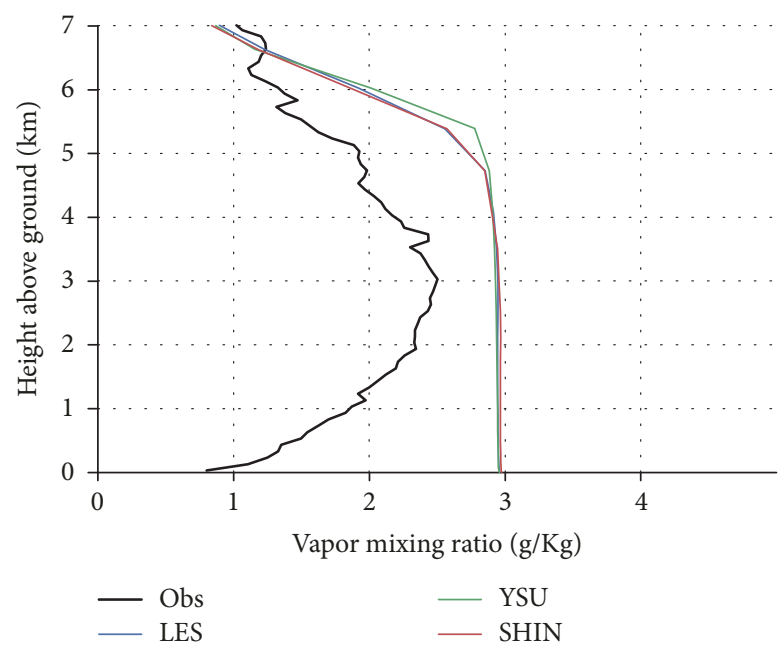

(d) 2000 BJT, 1 July 2016

FIgURE 3: The same as Figure 2, but for vapor mixing ratio.

correctly simulated the heating rate, but the simulated CBL were still relatively warmer than that observed. The potential temperature in the CBL appeared to be warmer by up to about $0.8 \mathrm{~K}$ in YSU, $0.6 \mathrm{~K}$ in Shin-Hong, and $0.52 \mathrm{~K}$ in WRF-LES, respectively, than the measurements at $1700 \mathrm{BJT}$.

The fundamental differences in moisture and temperature profiles between the Shin-Hong and YSU PBL schemes are illustrated in Figures 2 and 3. The profiles in the Shin-Hong experiment and the WRF-LES suggest the weak entrainment flux ratio, which was obviously overestimated in the YSU experiment. On the other hand, the moisture is more convergent and overestimated in the mixed layer compared to the measurements (Figure 3). Shin-Hong scheme also follows the WRF-LES well by the end of the day, except for the discrepancies in the surface layer (Figure 2(d)). The resulting thermal and moisture profiles from YSU and Shin-Hong PBL both show deviation from WRF-LES profiles. However, the profiles for the Shin-Hong scheme are closer to the WRF-LES profile than that of YSU, showing that ShinHong improves the simulations compared to YSU scheme because of the scale-dependent reference data introduced in Shin-Hong. It should be noted that results of WRF-LES (e.g., entrainment) are sensitive to horizontal grid spacing and vertical resolution [31]. WRF-LES is considered as a relatively trustable reference, because YSU and Shin-Hong PBL schemes used in this paper are both developed with the help of LES data.

Determining the calculation in PBL height $(h)$ is important in atmospheric numerical models, because $h$ is used in other physics parametrizations where required [5]. To find further discrepancies of the two nonlocal schemes in simulating Taklimakan desert CBL, PBL heights are calculated by the method in Liu and Liang [32] with four steps as follows:

(1) Observations and simulations are all interpolated to vertical profile with $50 \mathrm{~m}$ interval.

(2) The procedure starts with the regime identification by examining the near-surface thermal gradient between the fifth $(250 \mathrm{~m})$ and second levels $(100 \mathrm{~m})$, which are chosen specifically to remove the raw data noises. 


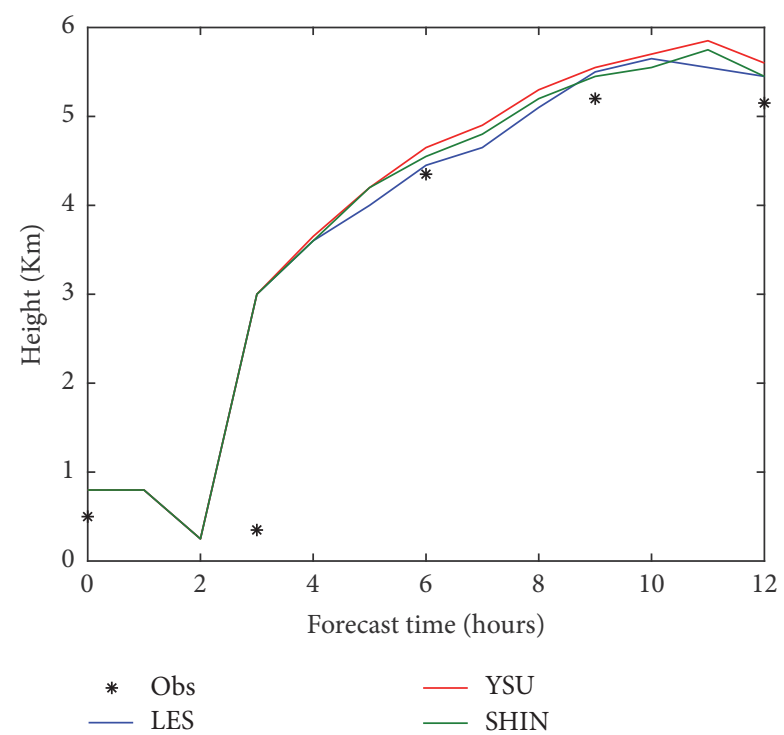

Figure 4: Time series of the PBL height (units: km) over Tazhong station from the GPS sounding (black), LES (green), YSU (red), and Shin-Hong (blue) experiments.

(3) Then, PBLH is determined as the height at which an air parcel rising adiabatically from the surface becomes neutrally buoyant. In practice, for the unstable regime, we first scan upward to find the lowest level.

(4) Then, this first-guess level $k$ is then corrected by another upward scan to search for the first occurrence of $\dot{\theta}_{k} / \partial z \geq \dot{\theta}_{r}$, where $\dot{\theta}_{k}$ is the $\theta$ vertical gradient per height $z$ and $\dot{\theta}_{r}$ is its minimum strength for the overlying inversion layer.

The empirical values of the three parameters $\left(\delta_{s}, \delta_{u}\right.$, and $\theta_{r}$ ) used in this method were proposed by Liu and Liang (2010) $\left(\delta_{s}=1.0 \mathrm{~K}, \delta_{u}=0.5 \mathrm{~K}\right.$, and $\left.\theta_{r}=4 \mathrm{~K} \mathrm{~km}^{-1}\right)$. Through our research, we found that the main uncertainty for the depth of the CBL in this study is parameter values $\theta_{r}$. Because the vertical gradient of potential temperature above mixed layer in all simulation experiments are weak relative to observations. Besides the performance of model simulation, another important reason for simulation results capped by a weak inversion is limited vertical resolution. It is hard to get the "detail" of the vertical gradient of potential temperature for model relative to observation. Therefore, we set parameter to $\theta_{r}$ smaller value $2 \mathrm{~K} \mathrm{~km}^{-1}$ to avoid overestimating the depth of entrainment zone for both observation and simulation results.

Results of PBL height from three experiments using the YSU PBL, WRF-LES, and Shin-Hong PBL schemes are presented in Figure 4. In response to the daytime variation of heat fluxes, all experiments which include WRF-LES simulate the growth of the mixed layer. The simulated height is nearly the same in the initial 3 hours' simulations. The YSU PBL simulates the highest $h$ after $0800 \mathrm{BJT}$ and until end of simulation in all simulations. In the Shin-Hong case, the maximum value of $5750 \mathrm{~m}$ is compared with the value

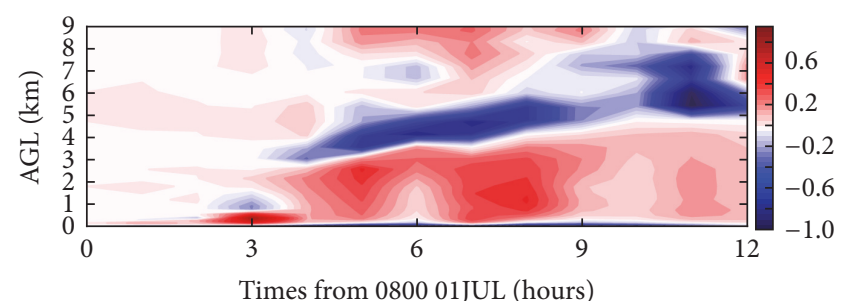

(a)

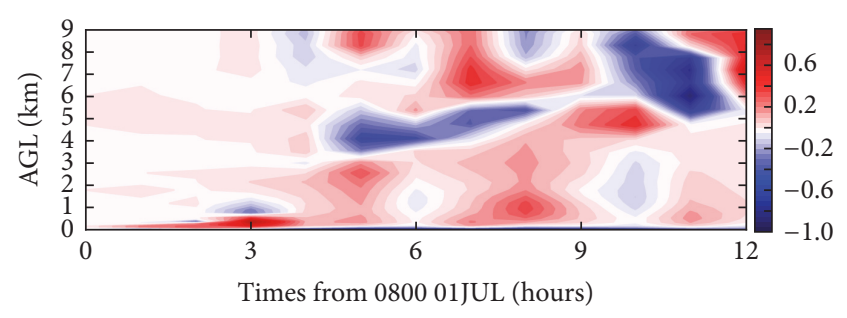

(b)

FIGURE 5: Time-height cross-section of the potential temperature differences at Tazhong station between (a) YSU, (b) Shin-Hong, and WRF-LES experiment, respectively.

of $5850 \mathrm{~m}$ in the YSU experiments. The YSU and ShinHong PBL scheme delay the growth of the maximum mixed layer by about an hour, with the maximum height bigger by 300 and $200 \mathrm{~m}$ than that from WRF-LES experiment (max: $5550 \mathrm{~m}$ ). Considering maximum value of $5200 \mathrm{~m}$ in observation, all experiments may overestimate PBL height and result in the warmer mixing layer in model simulations. The results show that Shin-Hong PBL schemes with scaleawareness simulate lower PBL than that in YSU PBL, which indicates overestimation of turbulent mixing in unstable stage of the boundary layer development over Taklimakan. the profiles in the CBL are cooler, drier, and more realistic in the Shin-Hong experiment similar to WRF-LES simulation, while the YSU PBL overestimates temperature and moisture of the profile in the CBL. The Shin-Hong PBL improves the modeled simulations compared to the YSU scheme.

In summary, the thermodynamic variables simulated with the two nonlocal PBL parametrizations are divergent before $1700 \mathrm{BJT}$, and Shin-Hong considering scale-aware is close to WRF-LES simulation in agreement with the observation. The variables are convergent and the values are close to the observations by the end of the day. On the other hand, the moisture profile experiences a similar impact on evolution with distinct deviations from the GPS sounding measurements. These results suggest that the scale-awareness in gray zone plays an important role in simulating the Taklimakan desert PBL, especially under unstable conditions. The ShinHong scheme shows better performance than convention nonlocal PBL scheme.

Figure 5 shows time-height cross-section of the potential temperature differences at Tazhong station between (a) YSU, (b) Shin-Hong, and WRF-LES experiments, respectively. The overall performance of the scale-awareness introduced in the Shin-Hong scheme can be better seen in Figure 5. Because of the reduced heating rate in the mixed layer and the enhanced 


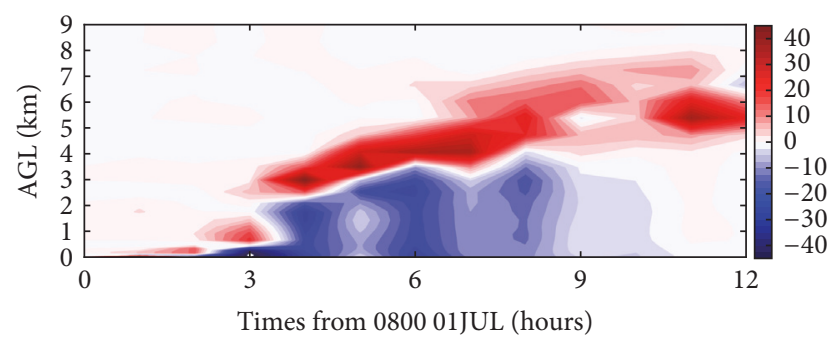

(a) YSU

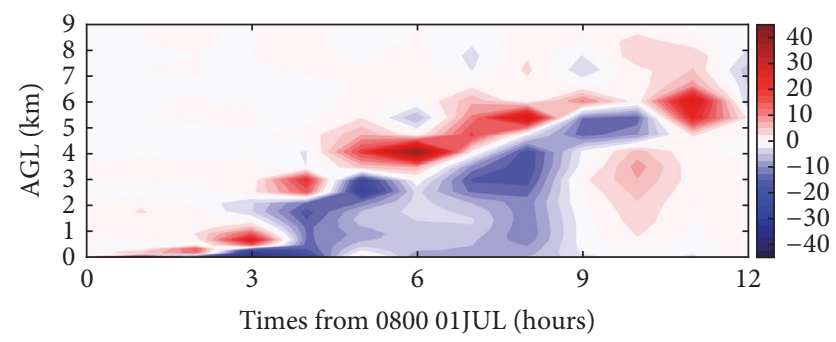

(b) SHIN

Figure 6: The same as Figure 5, but for vapor mixing ratio.

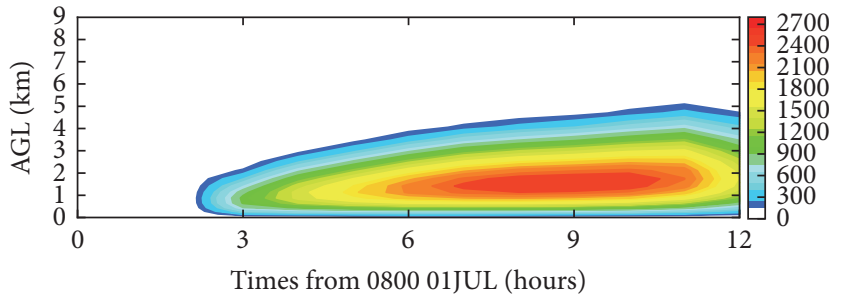

(a)

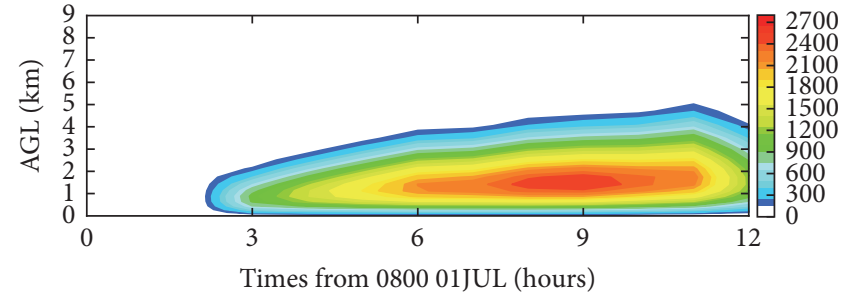

(b)

FIgURE 7: Time-height cross-section of the Eddy Viscosity km at Tazhong station from (a) YSU and (b) Shin-Hong experiments.

cooling rate in the entrainment layer, the Shin-Hong PBL scheme produces warmer temperatures near the PBL top $h$ and cooler in the mixed layer in agreement with WRF-LES (Figure 5(a)). The results further show that Shin-Hong PBL scheme reproduces much weaker entrainment layer than that of YSU as the mixed layer grows, which results in warming the entrainment layer and cooling the mixed layer (Figure 5(b)). On the other hand, in YSU PBL simulation, the rapid growth of PBL with strong vertical mixing in the PBL (Figure 4) results in the obvious decease of entrainment layer and the increase of mixing layer of the potential temperature. This is because of the improved nonlocal heat transport profile in Shin-Hong and the weaker strengths of vertical mixing in PBL as will be seen in Figure 7.

The vapor mixing ratio cross-section experiences a similar impact on evolution as that shown in the temperature (Figure 6). For the Shin-Hong scheme, a moister region is found under PBL height, whereas a drier PBL profile exists during the simulation period by the end of the day. The discrepancies of the moisture from YSU (Figure 6(a)) to WRF-LES experiment schemes are more noticeable than those of Shin-Hong (Figure 6(b)). Previous research (Xu et al., submitted) has examined the soil moisture for the Tazhong station. We quote the analysis of soil moisture for the Taklimakan area. In addition, the CTRL of Xu et al. is WRF-LES in this paper. At the model initialization for WRFLES simulation, EC station at Tazhong station indicated a value of the $5 \mathrm{~cm}$ deep soil moisture of $0.230 \mathrm{~m}^{3} / \mathrm{m}^{3}$, while the model initial value is $0.6 \mathrm{~m}^{3} / \mathrm{m}^{3}$. This large overestimate of soil moisture causes the $\mathrm{LH}$ from the model to continue increasing and then forces near-surface far moister than observation at the first few hours of model integration. However, an interesting result to note is that the model simulation has the abilities to correct some of the bias due to the initial condition of the surface.

We further examine the impact of scale dependencies on vertical mixing in the PBL over Taklimakan. Time-height cross-section of $K_{m}$ (vertical diffusivity for momentum) from the YSU and Shin-Hong PBL schemes over Tazhong station is depicted in Figure 7. Overall, the two schemes reveal a typical variation of local vertical mixing strengths in response to a diurnal variation of solar heating. The diffusivity vertical structures from the YSU and Shin-Hong experiments are similar. In the convective regime, the two experiments show maximum $1 \mathrm{~km}$ above the ground. However, the Shin-Hong scheme shows a weaker local vertical mixing than the YSU experiment. Since the two PBL schemes show very similar results in the Eddy Viscosity km, why Shin-Hong scheme can improve the profiles as showed above? In Shin and Hong (2015), one of the main improvements of the Shin-Hong PBL scheme is the prescribed nonlocal heat transport profile fitted to the WRF-LES output, in which cooling by the NL transport is limited to the surface layer. However, YSU does not conform to the LES output. As a result, it calculates cooling from the surface to the middle of the CBL. This results in the local component from the YSU model having the larger heating up to the middle of the CBL, while the amount of heating decreases above the surface layer in the WRF-LES.

4.2. Convective Cells. The simulation results discussed above indicate that the vertical processes of Taklimakan desert CBL are sensitive to scale dependencies in the nonlocal PBL sheme. The results of desert CBL profiles simulations agree with the previous studies for the CBL simulation over desert. In this section, the effects of dependency function on the evolution of desert deep $(>5000 \mathrm{~m})$ convection roll are 


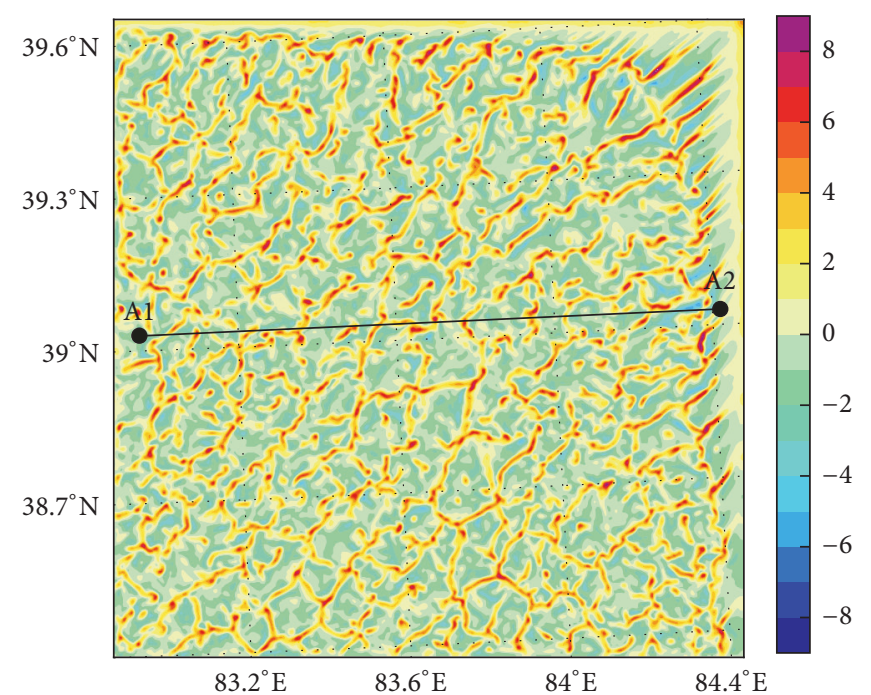

(a) Exp: LES time: 1400 BJT, 1 July 2016

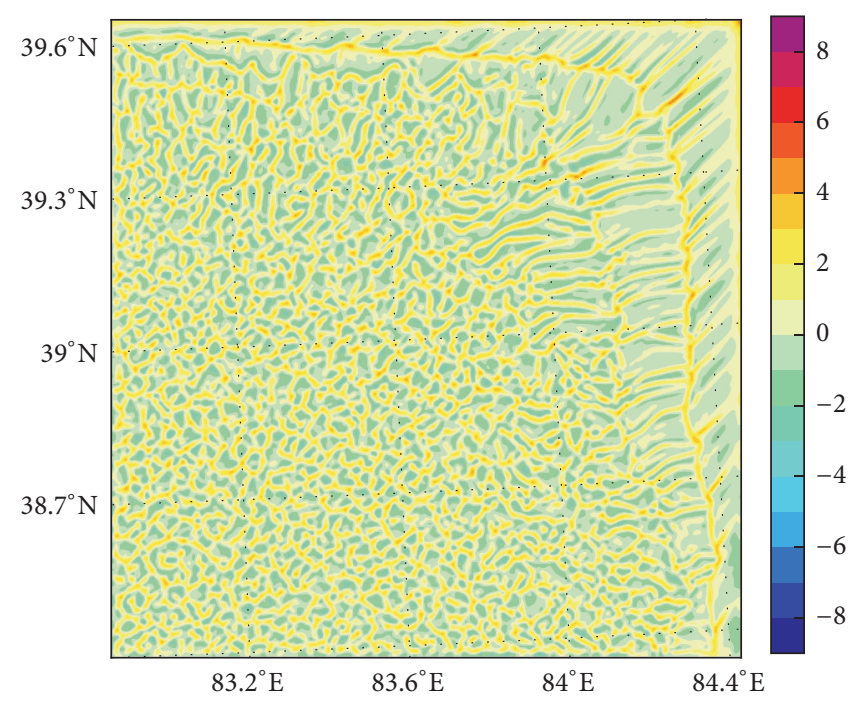

(b) Exp: YSU time: 1400 BJT, 1 July 2016

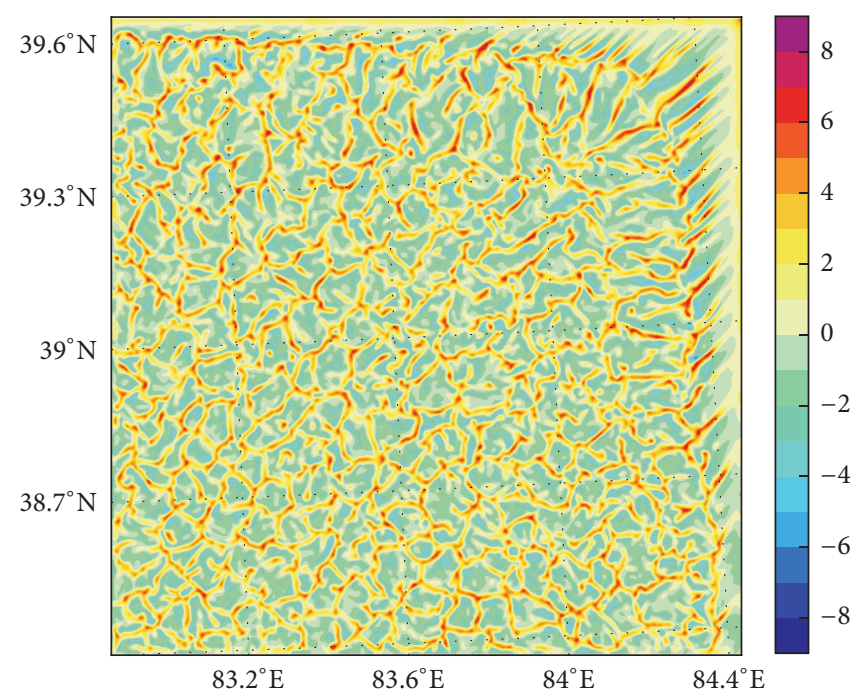

(c) Exp: SHIN time: 1400 BJT, 1 July 2016

FIGURE 8: Instantaneous vertical velocity fields (shading: $\mathrm{m} / \mathrm{s}$ ) at $3000 \mathrm{~m}$ for (a) WRF-LES, (b) YSU, and (c) Shin-Hong at 1400 BJT, 1 July 2016.

further examined and discussed. Specifically, we emphasize the impacts of the performance of two nonlocal PBL schemes on convection roll over gray zone scale.

The instantaneous vertical velocity fields for the horizontal are displayed in Figure 8. By 1400 BJT, the convection of WRF-LES simulation obviously intensified under strong surface heating. Thus, the maximum vertical velocity reaches $9 \mathrm{~m} / \mathrm{s}$ and the depth of mixed layer grows to about $4.3 \mathrm{~km}$ (Figure 8(a)). The distances between the boundary layer rolls correspondingly increase to about $12 \mathrm{~km}$ and the height of the peak updraughts is raised to just under $4 \mathrm{~km}$. The cellular shape of updraughts and downdraughts characteristic of boundary layer rolls is obvious in the horizontal view with the strength of convection. YSU PBL experiment reproduces motions with much weaker maximum and minimum values, due to excessive SGS heat transport (Figure 8(b)). In SHIN
PBL scheme, the excessive SGS heat transport in conventional nonlocal PBL schemes (such as YSU and ACM) has been suppressed and results in much stronger maximum and minimum value of $w$ (about $7 \mathrm{~m} / \mathrm{s}$ ). Furthermore, despite the underestimate of the peak value, the $w$ fields for the ShinHong experiment look similar to the WRF-LES $w$ in plain view, and the horizontal extent of the updrafts/downdrafts agrees with the WRF-LES as can be inferred from Figure 8. It was confirmed that Shin-Hong is a scale-aware PBL scheme in the sense of adjusting its resolved and subgrid-scale eddy with reference data from LES.

To further examine the impact of scale-dependent scheme on the simulation of desert CBL vertical cross-sections along Tazhong station $\left(39^{\circ} \mathrm{N}\right)$ of $w$ are presented in Figure 9. Wide and regularly spaced updrafts along A1A2 split into the stronger and more irregular motions in 


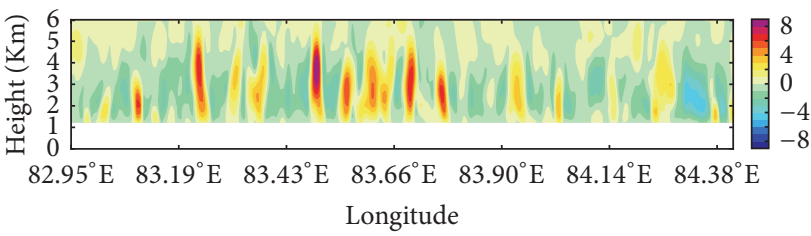

(a) Exp: LES time: 1400 BJT, 1 July 2016

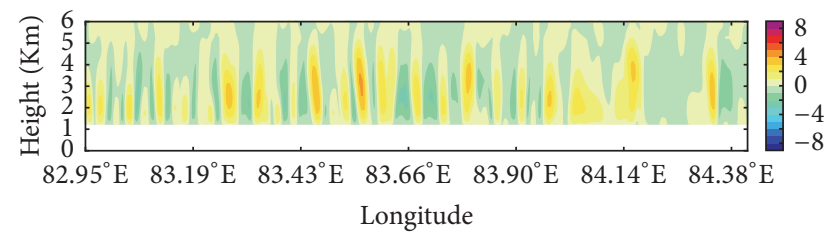

(b) Exp: YSU time: 1400 BJT, 1 July 2016

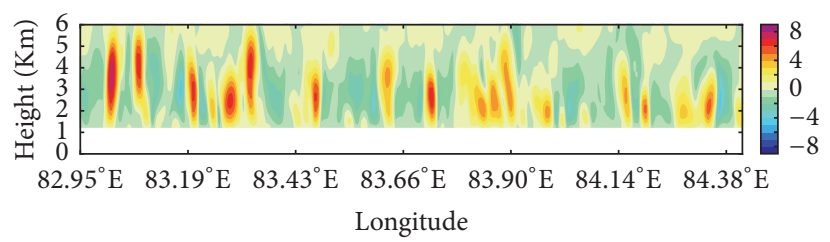

(c) Exp: SHIN time: 1400 BJT, 1 July 2016

FIGURE 9: Vertical cross-section of instantaneous vertical velocity fields (shading: $\mathrm{m} / \mathrm{s}$ ) along $\mathrm{A}_{1}-\mathrm{A}_{2}$ in for (a) WRF-LES, (b) YSU, and (c) Shin-Hong at 1400 BJT, 1 July 2016.

Shin-Hong PBL. The updrafts are much weaker and evenly distributed in the YSU experiment, as can be seen from Figure 9. Peak updrafts on YSU PBL are about $4 \mathrm{~m} / \mathrm{s} \mathrm{much}$ weaker than on Shin-Hong $(7.5 \mathrm{~m} / \mathrm{s})$ and WRF-LES $(9 \mathrm{~m} / \mathrm{s})$, and the horizontal distance between updraughts is much smaller. Overall, for YSU PBL, the scale of the downdraughts circulations is smaller, the form of convection is less cellular, and the intensity of the convection is weaker. The horizontal distribution of vertical velocity in YSU PBL experiments is relatively narrow. This indicates some degree of convection roll within the YSU PBL. Shin and Hong [4] suggest that the cellular convection that appeared at the YSU PBL is partly due to the PBL scheme used, while other major factors also influence the result. Overall, the good agreement between Shin-Hong and WRF-LES type of convective structure was encouraging, given that the use of conventional nonlocal scheme (YSU PBL) showed much larger discrepancies.

4.3. Energy Spectra and Horizontal Variances. Figure 10 shows power spectra of $w$ at $3000 \mathrm{~m}$ simulated on the d04 at 1400 BJT, 1 July 2016. Compared to Shin-Hong scheme, the YSU PBL substantially underestimates the resolved energy in the present case. The underestimate of the power spectra agrees quantitatively with the shape of the boundary layer rolls described above. Although the resolved energy is still slightly underestimated, this figure confirms the improvement in resolved motions by Shin-Hong PBL in the deep CBL of desert conditions. The Shin-Hong experiment shows an improvement over those from the YSU experiment compared with WRF-LES, but the energy is still weakly underestimated at almost all relevant scales.

To further examine the impact of effects of scale dependency in the PBL on the turbulence of Taklimakan desert boundary layer, the vertical profiles of domain-averaged resolved vertical heat transport are presented in Figure 11. It shows that the vertical gradient of $w$ decreases from surface to $500 \mathrm{~m}$ height, while the vertical gradient of $w$ increases from about 500 to $4000 \mathrm{~m}$ in each experiment over Tazhong station at $1400 \mathrm{BJT}$. The corresponding vertical

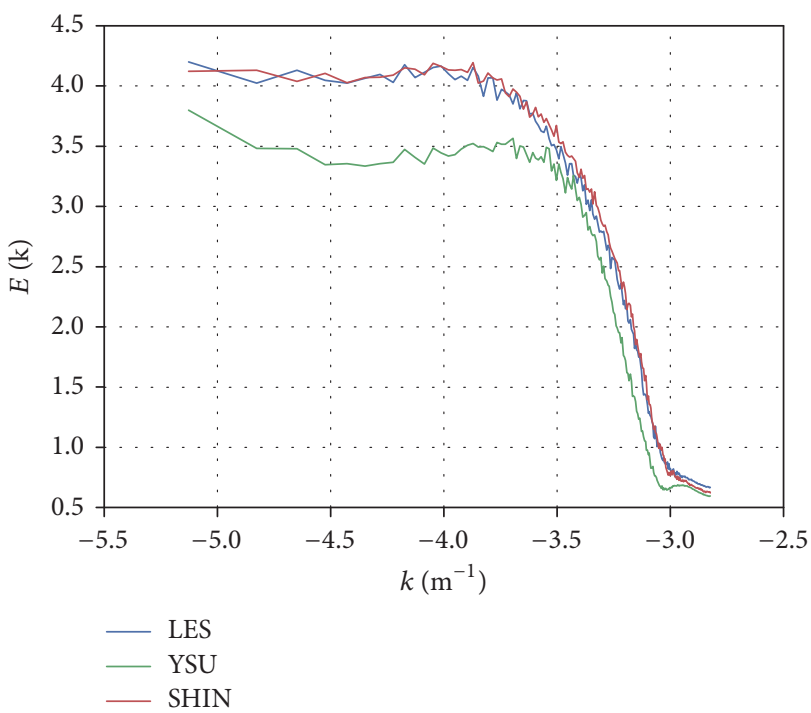

Figure 10: Power spectra of $w$ at $3000 \mathrm{~m}$ simulated on the d04 at 1400 BJT, 1 July 2016.

evolution of $w$ also shows a gradual decease and a gradient increase in lower and upper PBL, but much weaker than Shin-Hong and WRF-LES experiments. More importantly, the decease of lower PBL and the increase of upper PBL are most obvious in WRF-LES, followed by Shin-Hong resulting from correctly representing the strong convection roll, which is much weaker in YSU experiment. In other words, using explicit grid-size dependency functions derived by LES at gray zone resolutions by suppressing the role of SGS parameterization could correctly represent effects of scale dependency in Taklimakan desert CBL and increase vertical $w$ gradient in PBL in agreement with WRF-LES simulation. Shin and Hong [4] show that the advantage of Shin-Hong PBL is better representation of resolved fluxes. The resolved fluxes shown here are to confirm the applicability of Shin-Hong PBL in deep PBL height (>5000). However, the Shin-Hong 


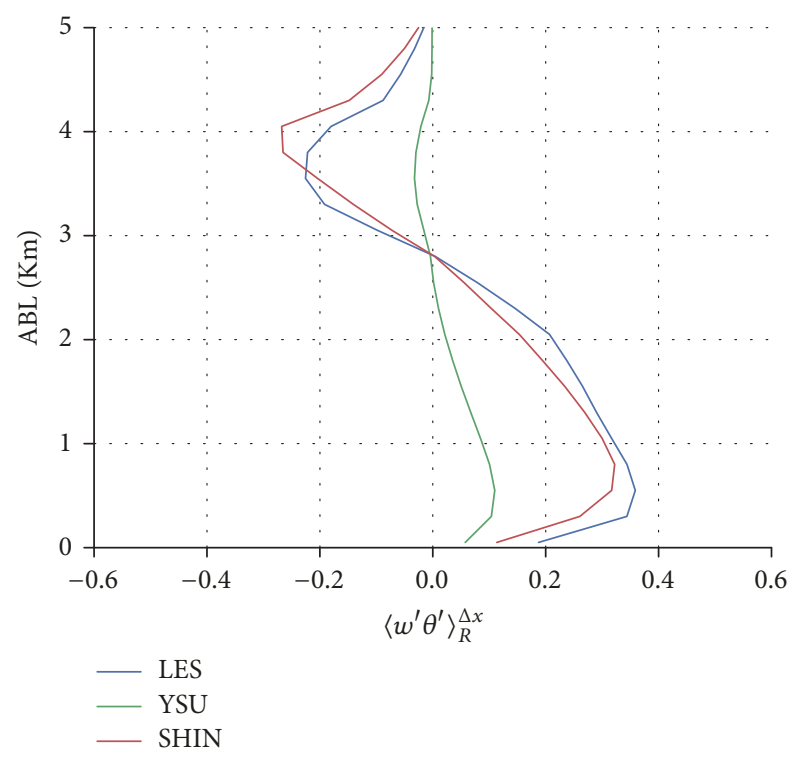

FIGURE 11: Vertical profiles of domain-averaged resolved vertical heat transport.

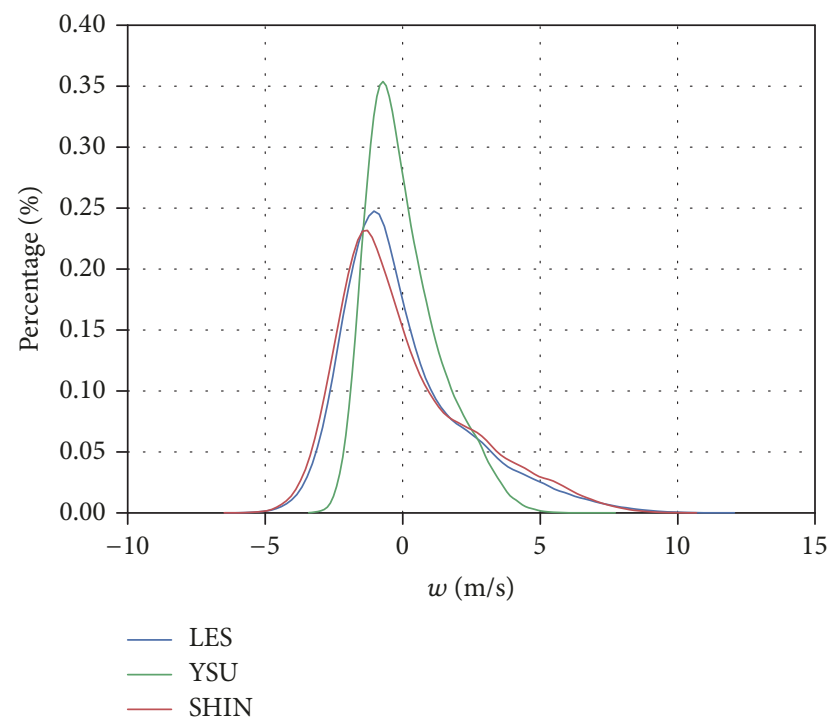

FIGURE 12: Probability distribution of vertical velocity from model simulation.

parameterization scheme has not been developed for extreme conditions like Taklimakan with super high PBL depth, where their assumptions and reference data from LES solutions of domains were only extended to $4000 \mathrm{~m}$ [17]. Direct applications of the scale-dependent PBL parameterizations to deep CBL $(>5000 \mathrm{~m})$ over desert such as Taklimakan still show better results than conventional scheme.

Probability distribution of vertical velocity from model simulation is presented in Figure 12. Result shows that the PDF for the Shin-Hong experiment is similar to the WRFLES. However, the PDF for Shin-Hong has slightly smaller values than the WRF-LES PDF for the strong updrafts or downdrafts. This confirms that in the scale-dependent reference data from LES contribute much to the simulation of strong vertical motions in nonlocal PBL scheme. On the other hand, the difference between the YSU and WRF-LES is large, with much weaker and less strong vertical velocity. This indicates that, in the YSU PBL, the strong vertical velocities are not mainly from $\mathrm{PBL}$ processes, while they are from PBL turbulence motions in the WRF-LES and Shin-Hong PBL.

\section{Discussion}

The results from the new algorithm Shin-Hong nonlocal PBL scheme with scale dependencies were evaluated against the field observation experiment of Tazhong station and compared with those from the conventional nonlocal, $\mathrm{K}$ profile YSU PBL scheme. The impact of scale dependencies in ARW-WRF on simulating Taklimakan desert deep CBL $(>5000 \mathrm{~m})$ is currently being tested. To deal with observational shortcomings, we simulate the Taklimakan desert with WRF-LES to implement the observation and further verify the performance of scale dependencies compared to conventional PBL scheme.

The results show that both nonlocal closure schemes are able to reproduce the typical three-layer potential temperature structure of the Taklimakan CBL, with a well-mixed CBL, weak inversion. Owing to the improved total nonlocal transport profile fit to the LES data, the simulated profiles in the CBL are cooler, drier, and more realistic in the Shin-Hong experiment than observation in agreement with WRF-LES simulation, while the YSU PBL overestimates temperature and moisture of the profile in the CBL. However, CBL growth in Shin-Hong is still slightly faster compared to the WRF-LES and still cannot represent temperature inversion in the morning and therefore smooths the lower CBL. This suggesting that there are still uncertainties, despite of using the state-of-the-art scale dependencies PBL scheme with reference data from LES. On the other hand, a representation of scale dependencies in the nonlocal PBL scheme is having a significant impact on simulations of Taklimakan desert CBL. As in Shin and Hong [17], the improvement in the profiles by the new algorithm, Shin-Hong, is mainly due to the "total" nonlocal transport profile fit to the LES data rather than to the inclusion of the grid-size dependency function, in that the improvement appears also at where the amount of the SGS transport is comparable to the total transport.

On the other hand, further analyses of CBL convection roll from this study show that the Shin-Hong PBL scheme is also relative advantage to YSU PBL scheme for simulating convective cell over Taklimakan deep CBL. Application of the Shin-Hong scheme to the convective-roll simulations forced by the analysis data confirmed that the roll structures simulated by the new algorithm with scale dependencies are more robust and the modeled circulations are stronger than the rolls produced by the conventional nonlocal PBL algorithm. The Shin-Hong simulation reproduces cellular convections roll with the magnitude slightly smaller than WRF-LES, and the conventional PBL scheme fails to trigger strong convective cell. Here we only provide a quick overview of potential problems in the representation of the Taklimakan CBL in submillimeter simulation given the insight 
provided by Tazhong field experiments and the WRF-LES results; a more in-depth analysis of these results will provide the basis for future work. In contrast to previous casestudies of deep desert CBL, for example, Engelstaedter et al. [33] found that the Met Office Unified Model, run as a single-column model, struggled to reproduce the observed organization of stratocumulus, highlighting the difficulties of PBL parameterization in reproducing key processes in the desert deep CBL. The present study indicates that the inclusion of scale dependencies of gray zone in model is very important even using the state-of-the-art conventional nonlocal PBL schemes [12]. In the present case, deep CBL structures over Taklimakan are sensitive to inclusion of scale dependency function in PBL parameterization. The revised total nonlocal transport profiles fit to the LES data improve the mean profiles. The grid-size dependency functions help the resolved motions and the resolved transport profiles improved via accurately computing the SGS transport profiles at different resolutions [4].

\section{Conclusion}

The results from Shin-Hong PBL scheme with scale dependency are encouraging in simulating deep CBL structures over Taklimakan and show better simulation of profile and convective cells compared to conventional nonlocal PBL scheme. The major conventional nonlocal scheme shortcomings-nonlocal transport profile and representation of the scale-can be dealt with in a straightforward way. While scale dependency scheme Shin-Hong is a useful metric for simulating deep desert CBL, uncertainties should also be considered. The mismatch between Shin-Hong and observation and WRF-LES is still present. This means that the uncertainties of gray zone in scale-dependent PBL scheme need to be further quantified. The model performance over Taklimakan still faces uncertainties due to the sparse data. More detailed data of Taklimakan (land and atmosphere) might be necessary to realize a more realistic performance.

\section{Conflicts of Interest}

The authors declare that there are no conflicts of interest regarding the publication of this paper.

\section{Acknowledgments}

This study is supported by the National Natural Science Foundation of China (Grants nos. 41575008 and 41775030).

\section{References}

[1] M. Wang, W. Wei, Q. He, Y. Yang, L. Fan, and J. Zhang, "Summer atmospheric boundary layer structure in the hinterland of Taklimakan Desert, China," Journal of Arid Land, vol. 8, no. 6, pp. 846-860, 2016.

[2] B. Zhou, J. S. Simon, and F. K. Chow, "The convective boundary layer in the terra incognita," Journal of the Atmospheric Sciences, vol. 71, no. 7, pp. 2545-2563, 2014.
[3] C. Talbot, E. Bou-Zeid, and J. Smith, "Nested mesoscale largeeddy simulations with WRF: Performance in real test cases," Journal of Hydrometeorology, vol. 13, no. 5, pp. 1421-1441, 2012.

[4] H. H. Shin and S.-Y. Hong, "Representation of the subgrid-scale turbulent transport in convective boundary layers at gray-zone resolutions," Monthly Weather Review, vol. 143, no. 1, pp. 250271, 2015.

[5] H. H. Shin and S.-Y. Hong, "Intercomparison of planetary boundary-layer parametrizations in the WRF model for a single day from CASES-99," Boundary Layer Meteorology, vol. 139, no. 2, pp. 261-281, 2011.

[6] H. H. Shin and J. Dudhia, "Evaluation of PBL parameterizations in WRF at subkilometer grid spacings: Turbulence statistics in the dry convective boundary layer," Monthly Weather Review, vol. 144, no. 3, pp. 1161-1177, 2016.

[7] E. Agee and A. Gluhovsky, "LES model sensitivities to domains, grids, and large-eddy timescales," Journal of the Atmospheric Sciences, vol. 56, no. 4, pp. 599-604, 1999.

[8] B. Heinold, P. Knippertz, and R. J. Beare, "Idealized large-eddy simulations of nocturnal low-level jets over subtropical desert regions and implications for dust-generating winds," Quarterly Journal of the Royal Meteorological Society, vol. 141, no. 690, pp. 1740-1752, 2015.

[9] B. Heinold, P. Knippertz, and J. H. Marsham, "Large Eddy Simulations of Nocturnal Low-Level Jets over Desert Regions and Implications for Dust Emission," in EGU General Assembly Conference, 2013.

[10] C.-H. Moeng, J. Dudhia, J. Klemp, and P. Sullivan, "Examining two-way grid nesting for large eddy simulation of the PBL using the WRF model," Monthly Weather Review, vol. 135, no. 6, pp. 2295-2311, 2007.

[11] S. Hong, Y. Noh, and J. Dudhia, "A new vertical diffusion package with an explicit treatment of entrainment processes," Monthly Weather Review, vol. 134, no. 9, pp. 2318-2341, 2006.

[12] X.-M. Hu, J. W. Nielsen-Gammon, and F. Zhang, "Evaluation of three planetary boundary layer schemes in the WRF model," Journal of Applied Meteorology and Climatology, vol. 49, no. 9, pp. 1831-1844, 2010.

[13] B. Xie, J. C. H. Fung, A. Chan, and A. Lau, "Evaluation of nonlocal and local planetary boundary layer schemes in the WRF model," Journal of Geophysical Research: Atmospheres, vol. 117, no. 12, Article ID D12103, 2012.

[14] J. Cuxart and M. A. Jimenez, "Deep radiation fog in a wide closed valley: study by numerical modeling and remote sensing," Pure and Applied Geophysics, vol. 169, pp. 911-926, 2012.

[15] R. Honnert, V. Masson, and F. Couvreux, "A diagnostic for evaluating the representation of turbulence in atmospheric models at the kilometric scale," Journal of the Atmospheric Sciences, vol. 68, no. 12, pp. 3112-3131, 2011.

[16] J. C. Wyngaard, "Toward numerical modeling in the "Terra Incognita", Journal of the Atmospheric Sciences, vol. 61, no. 14, pp. 1816-1826, 2004.

[17] H. H. Shin and S.-Y. Hong, "Analysis of resolved and parameterized vertical transports in convective boundary layers at grayzone resolutions," Journal of the Atmospheric Sciences, vol. 70, no. 10 , pp. $3248-3261,2013$.

[18] M. Z. Wang, H. Lu, H. Ming, and J. Zhang, "Vertical structure of summer clear-sky atmospheric boundary layer over the hinterland and southern margin of Taklamakan Desert," Meteorological Applications, vol. 23, no. 3, pp. 438-447, 2016. 
[19] Y. Liu, Q. He, H. Zhang, and A. Mamtimin, "Improving the CoLM in Taklimakan Desert hinterland with accurate key parameters and an appropriate parameterization scheme," Advances in Atmospheric Sciences, vol. 29, no. 2, pp. 381-390, 2012.

[20] W. C. Skamarock, J. B. Klemp, J. Dudhia et al., "A Description of the Advanced Research WRF Version 3., NCAR/TN475+STR," Tech. Rep., 2008.

[21] S.-Y. Hong and J.-O. J. Lim, "The WRF single-moment 6class microphysics scheme (WSM6)," Journal of the Korean Meteorological Society, vol. 42, no. 2, pp. 129-151, 2006.

[22] S.-Y. Hong and H.-L. Pan, "Nonlocal boundary layer vertical diffusion in a medium-range forecast model," Monthly Weather Review, vol. 124, no. 10, pp. 2322-2339, 1996.

[23] P. A. Jiménez, J. Dudhia, J. F. González-Rouco, J. Navarro, J. P. Montávez, and E. García-Bustamante, "A revised scheme for the WRF surface layer formulation," Monthly Weather Review, vol. 140, no. 3, pp. 898-918, 2012.

[24] J. S. Kain, "The Kain-Fritsch convective parameterization: an update," Journal of Applied Meteorology and Climatology, vol. 43, no. 1, pp. 170-181, 2004.

[25] J. S. Kain, "Convective parameterization for mesoscale models: The Kain-Fritsch scheme," in The representation of cumulus convection in numerical models, Meteor. Monogr, vol. 46, pp. 165-170, Springer, 1993.

[26] F. Chen and J. Dudhia, "Coupling an advanced land surface-hydrology model with the Penn State-NCAR MM5 modeling system. Part II: preliminary model validation," Monthly Weather Review, vol. 129, no. 4, pp. 587-604, 2001.

[27] F. Chen and J. Dudhia, "Coupling and advanced land surfacehydrology model with the Penn State-NCAR MM5 modeling system. Part I: model implementation and sensitivity," Monthly Weather Review, vol. 129, no. 4, pp. 569-585, 2001.

[28] E. J. Mlawer, S. J. Taubman, P. D. Brown, M. J. Iacono, and S. A. Clough, "Radiative transfer for inhomogeneous atmospheres: RRTM, a validated correlated-k model for the longwave," Journal of Geophysical Research: Atmospheres, vol. 102, no. 14, pp. 16663-16682, 1997.

[29] J. Dudhia, "Numerical study of convection observed during the Winter Monsoon Experiment using a mesoscale twodimensional model," Journal of the Atmospheric Sciences, vol. 46, no. 20, pp. 3077-3107, 1989.

[30] N. W. S. N. U. S. D. o. C. National Centers for Environmental Prediction, "NCEP GDAS/FNL 0.25 Degree Global Tropospheric Analyses and Forecast Grids," Ed., Research Data Archive at the National Center for Atmospheric Research, Computational and Information Systems Laboratory, Boulder, CO, 2015.

[31] C. S. Bretherton, M. K. Macvean, P. Bechtold et al., "An intercomparison of radiatively driven entrainment and turbulence in a smoke cloud, as simulated by different numerical models," Quarterly Journal of the Royal Meteorological Society, vol. 125, no. 554, pp. 391-423, 1999.

[32] S. Liu and X.-Z. Liang, "Observed diurnal cycle climatology of planetary boundary layer height," Journal of Climate, vol. 23, no. 21, pp. 5790-5809, 2010.

[33] S. Engelstaedter, R. Washington, C. Flamant, D. J. Parker, C. J. T. Allen, and M. C. Todd, "The Saharan heat low and moisture transport pathways in the central Sahara-Multiaircraft observations and Africa-LAM evaluation," Journal of Geophysical Research: Atmospheres, vol. 120, no. 10, pp. 4417-4442, 2015. 

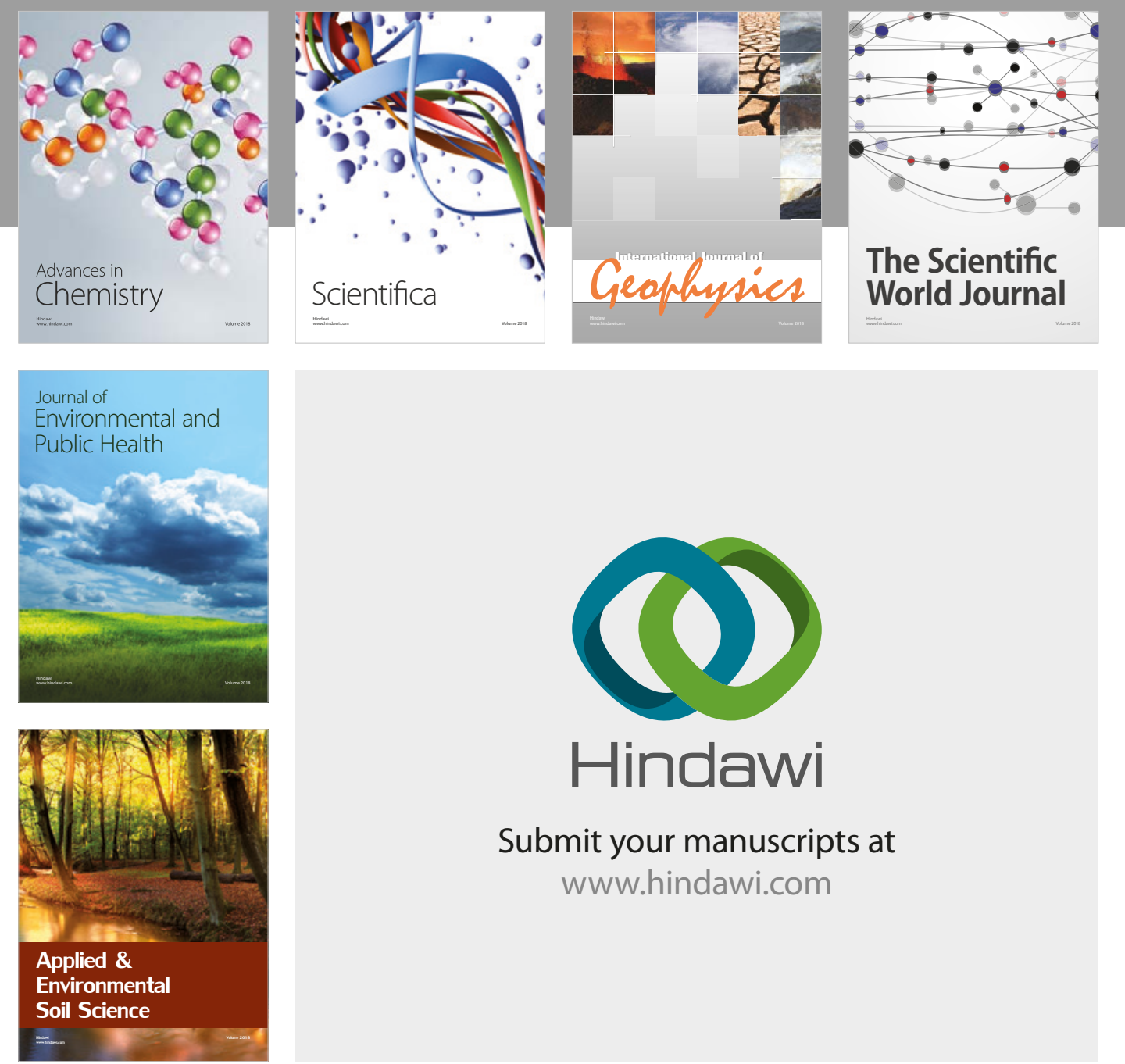

The Scientific

\section{World Journal}
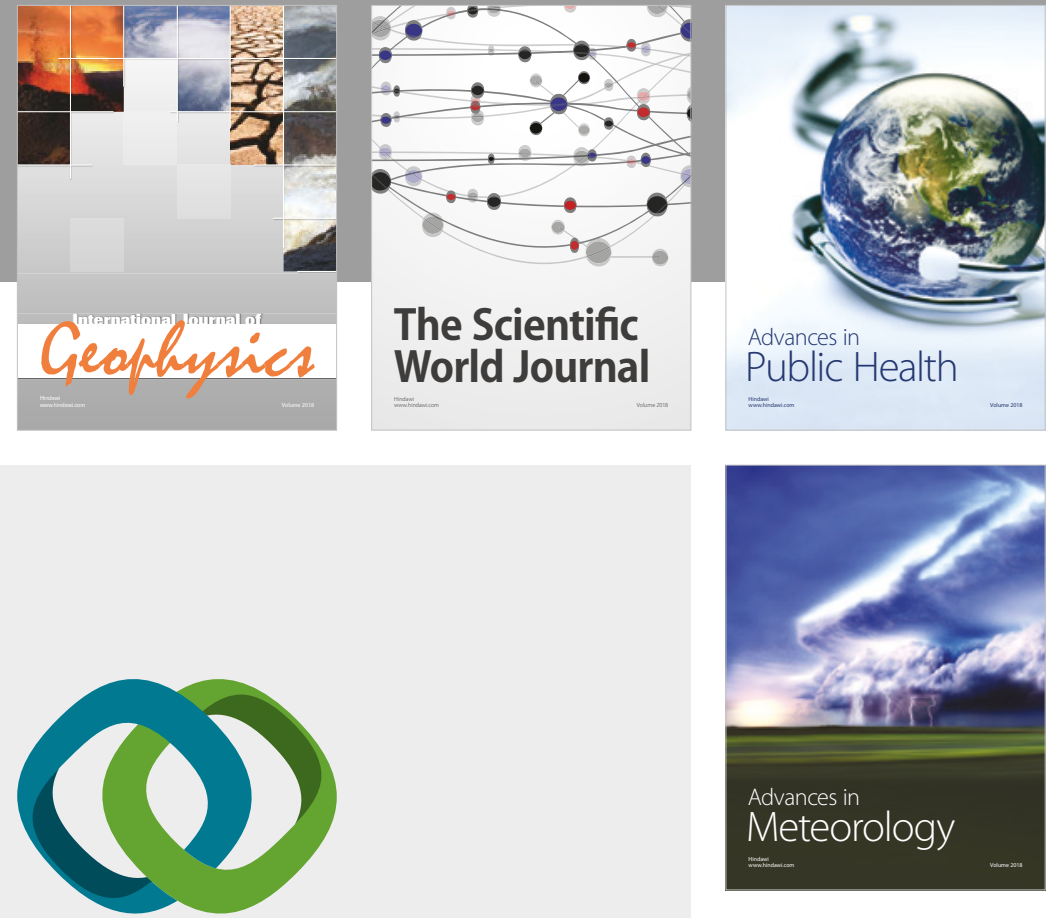

Advan

Public Health

\section{Hindawi}

Submit your manuscripts at

www.hindawi.com
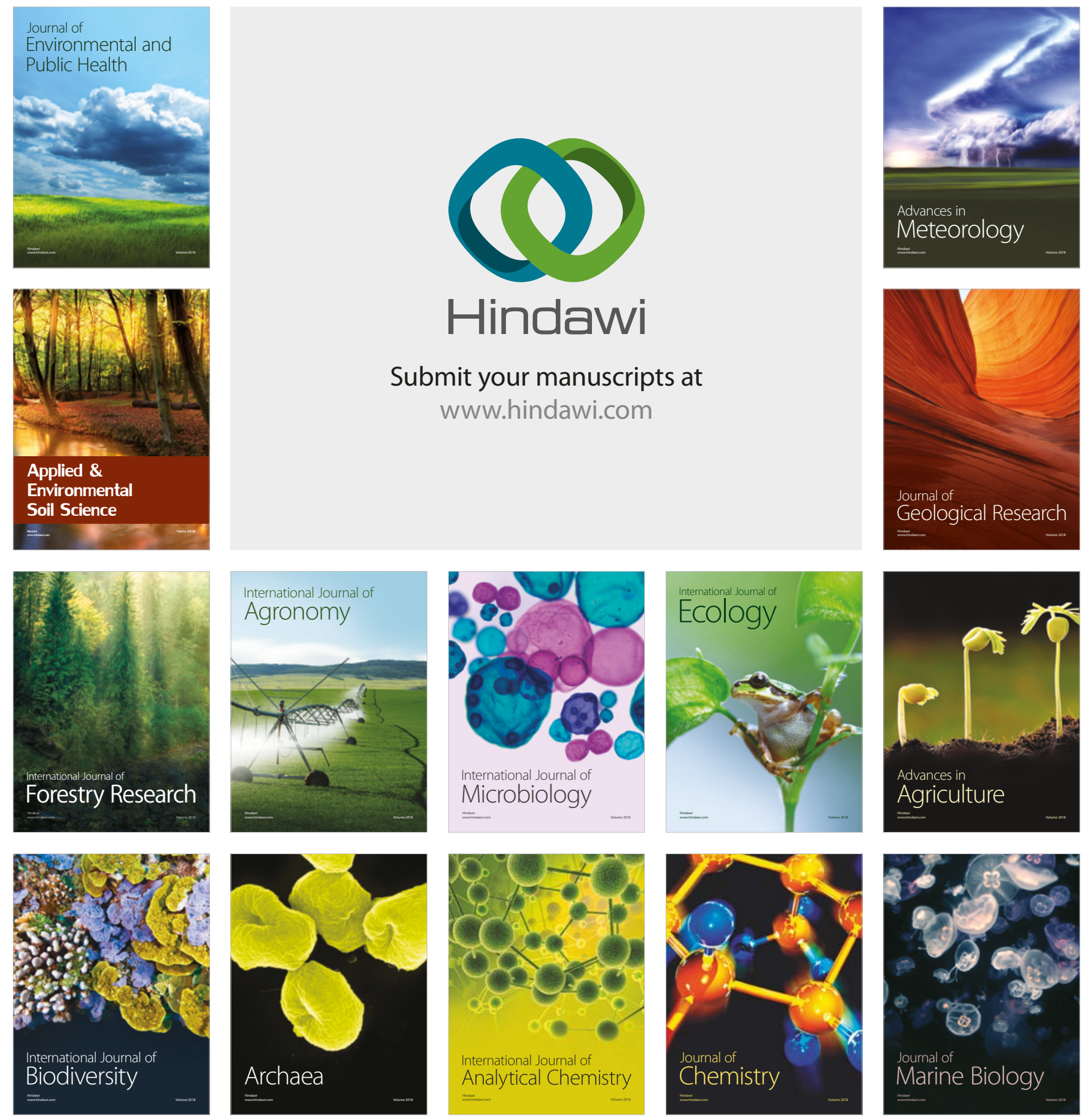